\title{
Genetic mutations in pfort and pfmdr1 at the time of artemisinin combination therapy introduction in South Pacific islands of Vanuatu and Solomon Islands
}

Karryn J Gresty ${ }^{1,2}$, Karen-Ann Gray ${ }^{1,2}$, Albino Bobogare ${ }^{3}$, George Taleo ${ }^{4}$, Jeffrey Hii ${ }^{5}$, Lyndes Wini ${ }^{3}$, Qin Cheng ${ }^{1,2}$ and Norman C Waters ${ }^{1,6^{*}}$

\begin{abstract}
Background: Chloroquine (CQ), alone or in combination with sulphadoxine-pyrimethamine, was widely used for the treatment of Plasmodium falciparum and Plasmodium vivax for several decades in both Vanuatu and Solomon Islands prior to the introduction of artemether-lumefantrine (AL) in 2008. However, the effect of chloroquine selection on parasite population, which may affect the efficacy of lumefantrine or other partner drugs of artemisinin, has not been well assessed. This study aims to provide baseline data on molecular markers (pfcrt and pfmdr1), along with the origins of pfcrt, prior to the introduction of AL.

Methods: Blood spots were obtained from epidemiological surveys conducted on Tanna Island, Tafea Province, Vanuatu and Temotu Province, Solomon Islands in 2008. Additional samples from Malaita Province, Solomon Islands were collected as part of an artemether-lumefantrine efficacy study in 2008. Plasmodium falciparum pfcrt and pfmdr 1 genes were examined for polymorphisms. Microsatellite markers flanking pfcrt were also examined to ascertain origins of CQ resistance.

Results: Pfcrt analysis revealed 100\% of parasites from Tafea Province, Vanuatu and Malaita Province, Solomon Islands and $98 \%$ of parasites from Temotu Province, Solomon Islands carried the K76T polymorphism that confers CQ resistance. Comparison of pfcrt allelic patterns and microsatellite markers flanking pfcrt revealed six haplotypes with more than $70 \%$ of isolates possessing haplotypes very similar to those observed in Papua New Guinea. The dominant (98.5\%) pfmdr1 allele across all island groups was YYCND.

Conclusions: Prior to the introduction of AL in the Solomon Islands and Vanuatu, P. falciparum isolates possessed point mutations known to confer CQ resistance and possibly associated with a decreased susceptibility to quinine and halofantrine, but an increased susceptibility to artemisinin and lumefantrine. Overall, pfcrt allelic types and the flanking microsatellite markers exhibited similarities to those of Papua New Guinea, suggesting these parasites share a common ancestry. The current use of AL for both $P$. falciparum and $P$. vivax infections will enable changes in these markers, in the absence of CQ pressure, to be monitored.
\end{abstract}

Keywords: Plasmodium falciparum, Chloroquine, pfcrt, Microsatellite markers, Surveillance, Molecular markers, Vanuatu, Solomon Islands

\footnotetext{
* Correspondence: norman.c.waters2.mil@mail.mil

${ }^{1}$ Australian Army Malaria Institute, Enoggera, Brisbane, Queensland, Australia

${ }^{6}$ Walter Reed Army Institute of Research, Malaria Vaccine Branch, Military

Malaria Research Program, Silver Spring, Maryland, USA

Full list of author information is available at the end of the article
} 


\section{Background}

Chloroquine-resistant (CQR) Plasmodium falciparum parasites arose in the late 1960s and have since spread throughout most malaria-endemic regions rendering chloroquine $(C Q)$ ineffective in many areas. The spread of $C Q$ resistance has been attributed to the resurgence of malaria morbidity and mortality during the 1990s [1,2]. In 2001, the World Health Organization (WHO) recommended the use of artemisinin-based combination therapy (ACT) for treatment of uncomplicated P. falciparum malaria [3]. Following this recommendation, the South Pacific countries of Vanuatu and Solomon Islands introduced ACT in 2008 [4].

CQ resistance in P. falciparum has been reported to associate with mutations in the parasite CQ resistance transporter gene ( $p f c r t$ ) located on chromosome 7 . Molecular studies demonstrated that resistance to CQ results from a series of mutations in $p f c r t$, of particular importance is the mutation causing a change from lysine (K) to threonine (T) at amino acid $76[5,6]$. The K76T and 11 other amino acids have been used as molecular markers for studying the evolution of CQ resistance. Various combinations of pfcrt mutations have been identified in different geographical locations and have been linked to past drug exposure [7].

Worldwide, there has been at least five major independent geographical origins of CQR pfcrt alleles. Initially four lineages were identified; one each from Indochina/Africa (CVIET) and Melanesia (SVMNT), and two from South America (SVMNT) [8-10]; a fifth lineage was later identified from the Philippines (C/SVMNT) [11,12]. Furthermore, SVMNT has also been linked with historical amodiaquine use [7]. The origins of various $p f c r t$ alleles have been mapped using microsatellite markers flanking pfcrt $[9,10,12]$. Microsatellites (MS) are short, simple, sequence repeats, and are prolific in the $P$. falciparum genome. When drug pressure exists in a parasite population, mutations that are advantageous to the parasite, for example those leading to drug resistance, are selected and propagated throughout the population. The MS markers that flank these resistance genes can also be propagated throughout the population via a form of genetic "hitchhiking' [9]. This leads to a reduction in the variation of allele frequency of the MS markers closest to the locus under selection [9].

Pfcrt mutations can also affect parasite susceptibilities to other anti-malarial drugs. For example, wild type pfcrt alleles confer a reduced susceptibility to lumefantrine, while mutant alleles can result in increased sensitivity to lumefantrine [13]. Transfection experiments have demonstrated the K76T mutation as the main determinant of $p f c r t$-dependent lumefantrine susceptibility [13]. Similarly, susceptibility levels of amodiaquine have been found to vary depending on the geographic origin of the mutant $p f c r t$ alleles [7].
The multidrug resistance gene ( $p f m d r 1$ ) coding for P-glycoprotein homologue-1 (pgh-1) located on chromosome 5 [14-16] was also implicated in CQ resistance. Mutations in pfmdr1 result in changes at amino acids 86, $184,1034,1042$, and 1246, and these changes have been associated with CQR in laboratory clones [14] although their associations with CQR were strain specific [15]. These polymorphisms in the pfmdr1 gene were also shown to affect parasite susceptibilities to structurally diverse antimalarial compounds, for example quinine, halofantrine, mefloquine, CQ, and artemisinin in vitro [15,17]. Additionally, an increase in copy number of the pfmdr1 gene was reported to play a role in reduced parasite susceptibility to artemisinin derivatives as well as mefloquine, halofantrine and quinine [18-20].

Both P. falciparum and Plasmodium vivax are endemic to the Solomon Islands and Vanuatu (except for the islands of Aneityum and Futuna which are malaria-free). CQ was used as first-line therapy, alone or with sulphadoxinepyrimethamine (SP), for both $P$. falciparum and $P$. vivax infections until 2008. Reduced susceptibility of $P$. falciparum parasites to CQ was first described in the Solomon Islands in 1980, initially occurring in Western, Central, Guadalcanal, and Malaita Provinces, extending countrywide in later years [21,22]. In 2001, as the 28-day CQ treatment failure rate reached 67\% [22] Solomon Islands replaced CQ with CQ plus SP as first-line therapy for uncomplicated falciparum malaria. In Vanuatu, resistance to CQ was first reported in 1987, following this the Vanuatu Ministry of Health introduced a new treatment regime in 1994, changing the treatment of uncomplicated $P$. falciparum infection from CQ monotherapy to a combination therapy of $C Q$ and $S P$, while $C Q$ remained the treatment for $P$. vivax infections [22]. A therapeutic efficacy study conducted in 2001 revealed a 16\% treatment failure rate for CQ plus SP [22]. Despite these clinical reports, there is limited molecular data published on the prevalence of CQR malaria in Vanuatu and the Solomon Islands and their origins.

Vanuatu and the Solomon Islands are presently intensifying malaria control countrywide and progressing towards malaria elimination in targeted provinces. The success of eradicating malaria is dependent on many factors, not least of which is overcoming the challenge of drug resistance; malaria epidemiology is an integral component of any elimination strategy. Knowledge of baseline drug-resistant genotypes and their changes over time in parasite populations is critical to monitoring emergence of drug resistance and informing treatment policy. In 2008 artemether-lumefantrine (AL) was introduced to treat uncomplicated $P$. falciparum infections in both Vanuatu and the Solomon Islands. ACT is currently the first-line treatment for vivax malaria in both countries [4]. It is important to document parasite molecular drug resistance 
profiles at the time of AL introduction and use this baseline information for detection of future changes under $\mathrm{AL}$ pressure. Furthermore, the baseline information may also inform parasite susceptibility to companion drugs of ACT.

In this study, the occurrence and prevalence of mutations in $p f c r t$ and $p f m d r 1$ was examined. Samples used in this study were collected from villages across several isolated groups of islands from Vanuatu and the Solomon Islands in 2008. The origins of CQ resistance was also investigated by analysing the MS loci surrounding the pfcrt gene.

\section{Methods}

\section{Study areas and sample collection}

Samples used in this paper were collected in three separate studies. Two sets were obtained from epidemiological surveys conducted (2008) during the wet seasons in:

1. Tafea Province, Vanuatu. This was a school-based, mass blood survey of children (2-12 years) where $73 \%$ of all children both febrile and non-febrile were assessed for malaria infection.

2. Temotu Province, Solomon Islands. This was a village-based mass blood survey encompassing all ages where $50.2 \%$ of the provincial population both febrile and non-febrile were assessed for malaria infection.

The geographic locations and information regarding the demographics of the populations surveyed and consent process for both these surveys have been published previously [23].

The third set of samples was from an AL (Coartem ${ }^{\mathrm{TM}}$, Novartis) therapeutic efficacy study conducted (June to October 2008) in the Province of Malaita, Solomon Islands (Wini et al., unpublished). Febrile patients (2-68 years) attending Auki town clinic and Kilu'Ufi General Hospital were recruited by the Ministry of Health staff, Solomon Islands in collaboration with WHO. Malaita Province lies approximately $9^{\circ} \mathrm{S} 161^{\circ} \mathrm{E}$ and is the second largest and most densely populated Province of the Solomon Islands. In these areas malaria transmission is perennial with peaks between November and August.

Across all study sites blood samples were collected by finger prick. A blood spot $(20-30 \mu \mathrm{L})$ was air-dried onto filter paper (Whatman No 3), sealed in individual plastic bags containing desiccant and stored at room temperature until further processing.

\section{Parasite DNA extraction and PCR speciation}

Extraction of genomic DNA from blood on filter paper and PCR to determine Plasmodium species has been described previously [23].

\section{Parasite speciation by multiplex PCR}

Plasmodium speciation was performed using speciesspecific primers in a multiplex PCR as previously described [24] to identify P. falciparum, P. vivax, Plasmodium ovale or Plasmodium malariae infections in blood samples.

\section{Mutations in pfort}

The extracted DNA was used as the template for PCR amplification of pfcrt. Initially PCR/RFLP analysis [25] was used to determine isolates exhibiting the K76T substitution indicative of CQ resistance. Random subsets of these samples (17 from Tafea, 50 from Temotu and 31 from Malaita Provinces) were selected for further analysis of additional codons. DNA fragments covering 12 known mutations in pfcrt were amplified by nested PCR as described previously [26]. For Tafea and Temotu Provinces, in addition to codon 76, 11 other codons were examined (72, 74, 75, 97, 144, 160, 220, 271, 326, 356, and 371), only four additional codons (72, 74, 75, and 97) were determined for samples from Malaita Province.

\section{Mutations in Pfmdr1}

Random subsets of samples (17 from Tafea, 37 from Temotu and 31 from Malaita Provinces) were selected for sequence analysis. The extracted DNA was used as the template for PCR amplification of DNA fragments covering five amino acid codons in pfmdr1 (86, 184, 1034,1042 , and 1246) known to affect drug resistance levels as described previously [27].

\section{DNA sequencing}

All PCR amplifications generating a single product were incubated with ExoSAP-IT ${ }^{\mathrm{R}}$ (USB, Cleveland, OH, USA) to remove excess primers and nucleotides according to the manufacturer's protocol and then sequenced. In cases where PCR products contained multiple bands, a single band of expected size was excised and purified using a NucleoSpin $^{\mathrm{R}}$ extraction kit (Macherey-Nagel) and then sequenced. Sequencing was performed using a Big Dye Terminator kit (v.3.1) on an automated DNA sequencer, ABI 3100 system, at the QIMR Berghofer Medical Research Institute Scientific Services Analytical Facility.

\section{Plasmodium falciparum microsatellite genotyping}

For selected samples five MS markers flanking the $p f c r t$ gene, B5M77 (-20 kb), 2E10 (-5 kb), PE12A (+6 kb), 2H4 $(+22 \mathrm{~kb})$, and PE14F (+106 kb), were amplified and analysed as described previously [9]. An additional six P. falciparum laboratory isolates (AN001, AN018, Dd2, $\mathrm{C} 2 \mathrm{~B}, \mathrm{PH} 1$, and 7G8) obtained from diverse geographical regions were analysed as reference strains [12]. MS sizes in samples were compared to these reference strains and calibrated accordingly. 


\section{Results}

Prevalence of mutations in pfcrt

Sequences of $p f c r t$ were examined and the prevalence of mutations determined for the three island groups. Amino acid substitutions were identified at codons 72, $76,220,326$, and 356 whereas wild type amino acids were observed at codons 97, 144 and 160. Initial PCR/ RFLP analysis revealed 100\% of parasites from Tafea Province, Vanuatu and Malaita Province, Solomon Islands and $98 \%$ of parasites from Temotu Province, Solomon Islands carried the K76T substitution indicative of CQR (Table 1). Only one sample from the village of Neo on Santa Cruz Island, Temotu Province exhibited the wild type lysine $(\mathrm{K})$ at codon 76 as well as cysteine $(\mathrm{C})$ at codon 72, however this sample had the A220S substitution (Table 2). All three island groups possessed pfcrt C72S and K76T substitutions at 94 and $98 \%$ or greater, respectively. Likewise, polymorphisms A220S, N326D/S and 356 L/T were $91 \%$ or greater for Tafea and Temotu Provinces (these codons were not examined for Malaita Province). All other codons examined indicated predominance for wild type amino acids M74, N75, H97, A144, L160, Q271, and R371 (Table 1).

\section{Pfcrt allelic types}

Sequence analysis of pfcrt at codons $72,74,75,76$, and 97 were completed for all samples investigated (17 from Tafea, 50 from Temotu and 31 from Malaita Provinces) and results revealed that $96 / 98$ (98\%) to be allelic type SMNTH. One other mutant allelic type CIETH was identified in a sample from Vanuatu while a wild type allele CMNKH was identified in one sample from Temotu Province, Solomon Islands.

In conjunction with these five codons, additional PfCRT amino acids 144, 160, 220, 271, 326, 356, and 371 were examined in samples from Tafea Province, Vanuatu and Temotu Province, Solomon Islands. Amino acids were determined at all 12 codons investigated for 48 isolates from Tafea Province, Vanuatu and Temotu Province, Solomon Islands, with remaining isolates having incomplete amino acid identification at one or more codons. Of the 51 completed sequences a predominant allele, SMNTHALSQDLR, was identified in 38 isolates
(74.5\%), with the remainder being predominantly polymorphic at codons 271, 326 and 356. This predominant allele was found in 83\% (5/6) and 73\% (33/45) of samples from Tafea Province, Vanuatu and Temotu Province, Solomon Islands, respectively. One sample from Port Resolution on Tanna Island, Tafea Province possessed allele CIETHALSE*TR (unidentified amino acids are represented by asterisk) similar to the dominant allele in samples originating in Thailand. In Tafea Province, Vanuatu, 33\% (3/9) of samples possessed the Q271E mutation most commonly seen in Thailand. Interestingly, this mutation was not detected in Temotu Province, Solomon Islands despite twice the number of samples being examined. Furthermore, one CQR isolate (SMNTH $H^{* *} \mathrm{~A}^{* * \mathbf{T} R}$ ) from the village of Ngamubulo, situated on the Reef Islands, Temotu Province, Solomon Islands did not exhibit the A220S polymorphism generally associated with CQR alleles. Observed allelic types and their prevalence in the different island groups are summarized in Table 2.

\section{Microsatellite haplotypes}

Throughout the island provinces, genotyping of five MS markers flanking $p f c r t$ revealed six haplotypes (based on similarity of 4/5 markers), H1-H6. The prevalence of each haplotype is different between the island groups (Table 2 and Table 3).

In Temotu Province, Solomon Islands, 34 of the 50 samples were successfully typed at all five loci and resulted in three haplotypes, of these $76.5 \%(26 / 34)$ and $20.6 \%$ (7/34) were $\mathrm{H} 3$ and $\mathrm{H} 4$, respectively, representing $97.1 \%$ of the parasite population (Table 3). These haplotypes have been reported in PNG (AN018 and AN001 groups). Interestingly, one sample from the village of Neo on Santa Cruz Island possessing a single A220S polymorphism, exhibited the Southeast Asian pfcrt allelic type and also had a MS pattern matching that of the Thailand C2B group at all three downstream MS loci.

In Malaita Province, Solomon Islands, 28 of the 31 isolates were successfully typed at all five loci and revealed four MS haplotypes (based on similarity of 4/5 MS markers). Of these $71.4(20 / 28)$ and $14.3 \%(4 / 28)$ were H3 and $\mathrm{H} 4$, respectively, representing $85.7 \%$ of parasite population (Table 3). Three samples were classified as H1,

Table 1 Prevalence (\%) of amino acid substitutions in PfCRT in Plasmodium falciparum samples collected from Tafea Province, Vanuatu and Temotu and Malaita Provinces, Solomon Islands

\begin{tabular}{|c|c|c|c|c|c|c|c|c|c|c|c|c|c|c|}
\hline \multirow[t]{2}{*}{ Country } & \multirow[t]{2}{*}{ Province } & \multirow[t]{2}{*}{$\mathrm{n}$} & \multicolumn{12}{|c|}{ \% substitutions at amino acid positions in PfCRT } \\
\hline & & & 72 & 74 & 75 & 76 & 97 & 144 & 160 & 220 & 271 & 326 & 356 & 371 \\
\hline Vanuatu & Tafea & 17 & 94 & 6 & 6 & 100 & 0 & 0 & 0 & 100 & 43 & 100 & 94 & 47 \\
\hline \multirow[t]{2}{*}{ Solomon Islands } & Temotu & 50 & 98 & 0 & 0 & 98 & 0 & 0 & 0 & 98 & 0 & 91 & 98 & 22 \\
\hline & Malaita & 31 & 100 & 0 & 0 & 100 & 0 & * & * & * & * & * & * & $*$ \\
\hline
\end{tabular}


Table 2 Pfmdr1 and pfcrt genotypes and MS markers flanking pfcrt in Plasmodium falciparum samples collected from Tafea Province, Vanuatu and Temotu and Malaita Provinces, Solomon Islands compared to reference samples

\begin{tabular}{|c|c|c|c|c|c|c|c|c|c|c|}
\hline \multirow[t]{2}{*}{ Origin (no. isolates) } & \multirow[t]{2}{*}{$\mathbf{n}$} & \multirow[t]{2}{*}{$\mathrm{H}$} & \multirow[t]{2}{*}{$\%$} & \multicolumn{2}{|c|}{$\mathrm{MS}^{\mathrm{a}}$ marker size (bp) } & \multirow{2}{*}{$\begin{array}{l}\text { AA positions } \\
\text { in } \mathrm{PfCrt}^{\mathrm{b}}\end{array}$} & \multicolumn{3}{|c|}{$M^{c}{ }^{c}$ marker size (bp) } & \multirow{2}{*}{$\begin{array}{l}\text { AA positions } \\
\text { in } P f m d r 1^{d}\end{array}$} \\
\hline & & & & B5M77 & $2 \mathrm{E} 10$ & & PE12A & $2 \mathrm{H} 4$ & PE14F & \\
\hline \multirow[t]{8}{*}{ Vanuatu Tafea $(n=17)$} & 5 & 1 & 29.4 & 147 & 174 & SMNTHALSQDLR & 328 & 184 & 142 & YYCND \\
\hline & 1 & 1 & 5.9 & 147 & 174 & SMNTHALSQDLI & 314 & 184 & 142 & YYCND \\
\hline & 1 & & 5.9 & * & * & SMNTHALS $\mathbf{S}^{* * *} \mathrm{R}$ & * & * & * & YYCND \\
\hline & 2 & 2 & 11.7 & 157 & 156 & SMNTHALSE*TI & * & 201 & 148 & $Y F^{*} N D$ \\
\hline & 5 & & 29.4 & * & * & SMNTHALS ${ }^{* *} \mathrm{TI}$ & * & * & * & YYCND \\
\hline & 1 & & 5.9 & * & * & SMNTHALS $\mathbf{S}^{* *}$ TR & * & * & $*$ & YYCND \\
\hline & 1 & & 5.9 & * & * & SMNTHALS*STR & * & * & * & YYCND \\
\hline & 1 & & 5.9 & * & * & CIETHALSE*TR & * & $*$ & $*$ & YYCND \\
\hline \multirow[t]{17}{*}{ Solomon Islands Temotu $(n=50)$} & 23 & 3 & 64.7 & 149 & 174 & SMNTHALSQDLR & 328 & 192 & 139 & YYCND \\
\hline & 4 & 4 & 8.9 & 149 & 174 & SMNTHALSQDLR & 328 & 184 & 134 & YYCND \\
\hline & 1 & 4 & 2.9 & 149 & 174 & SMNTHALSQDLR & 328 & 184 & 136 & YYCND \\
\hline & 1 & 4 & 2.9 & 149 & 174 & SMNTHALSQDLR & 328 & 184 & 142 & YYCND \\
\hline & 1 & & 2.9 & * & * & SMNTHALSQDLR & * & * & * & YYCND \\
\hline & 1 & 4 & 33.3 & 149 & 174 & SMNTHALSQDTR & 328 & 184 & 134 & YYCND \\
\hline & 1 & & 2.9 & * & * & SMNTHALSQDTR & * & * & * & ${ }^{*} \mathrm{CND}$ \\
\hline & 7 & & 5.9 & * & * & SMNTHALSQSTI & * & * & * & ${ }^{*} \mathrm{CND}$ \\
\hline & 2 & & 5.9 & $*$ & * & SMNTHALSQ*TI & * & * & * & ${ }^{*}{ }^{*} \mathrm{CND}$ \\
\hline & 1 & 5 & 2.9 & 140 & 166 & CMNKHALSQNIR & 314 & 184 & 148 & ${ }^{* *} \mathrm{CND}$ \\
\hline & 2 & 3 & 28.5 & 149 & 174 & SMNTHALSQDLR & 328 & 192 & 139 & YYCND \\
\hline & 1 & 3 & 14.2 & 149 & 174 & SMNTHALSQDLI & 328 & 192 & 139 & YYCND \\
\hline & 1 & & 14.2 & * & * & SMNTHALSQDLR & * & * & $*$ & YYCND \\
\hline & 1 & & 16.7 & * & * & SMNTHALSQSLR & * & * & * & ${ }^{* *} \mathrm{CND}$ \\
\hline & 1 & & 14.2 & * & * & SMNTHAL ${ }^{* *} \mathbf{S T R}$ & * & * & $*$ & YYCND \\
\hline & 1 & & 14.2 & * & * & SMNTHAL**STI & * & * & * & YYCND \\
\hline & 1 & & 14.2 & $*$ & * & $\mathbf{S M N T H} H^{* *} A^{* *} \mathbf{T} R$ & * & * & $*$ & ${ }^{* *} \mathrm{CND}$ \\
\hline \multirow[t]{6}{*}{ Solomon Islands Malaita $(n=31)$} & 9 & 3 & 29 & 149 & 174 & SMNTHALSQDLR & 328 & 192 & 139 & YYCND \\
\hline & 11 & 3 & 35.5 & 149 & 174 & SMNTHALSQDLI & 260 & 192 & 139 & YYCND \\
\hline & 4 & 4 & 12.9 & 149 & 174 & SMNTHALS $\mathbf{S}^{* * *} R$ & 328 & 184 & 145 & YYCND \\
\hline & 1 & 6 & 3.2 & 149 & 154 & SMNTHALSE*TI & 328 & 192 & 142 & YYCND \\
\hline & 3 & 1 & 9.7 & 147 & 174 & SMNTHALS S*TI & 328 & 184 & 142 & YYCND \\
\hline & 3 & & 9.7 & * & * & SMNTHALS $\mathbf{S}^{* *} \mathbf{T R}$ & * & * & * & YYCND \\
\hline \multirow[t]{7}{*}{ World reference Samples } & \multicolumn{3}{|c|}{ Brazil 7G8 } & 151 & 190 & SMNTHALSQDLR & 314 & 194 & 142 & \\
\hline & \multicolumn{3}{|c|}{ Philippines PH1 } & 149 & 182 & CMNTHTYAQDLR & 314 & 228 & 136 & \\
\hline & \multicolumn{3}{|c|}{ Thailand Dd2 } & 149 & 170 & CIETHALSESTI & 314 & 204 & 145 & \\
\hline & \multicolumn{3}{|c|}{ Thailand C2B } & 149 & 170 & CIETHALSESTI & 314 & 184 & 148 & \\
\hline & \multicolumn{3}{|c|}{ Solomon Island N18 } & 149 & 174 & SMNTHALSQDLR & 328 & 184 & 142 & \\
\hline & \multicolumn{3}{|c|}{ PNG AN001 } & 149 & 174 & SMNTHALSQDLR & 328 & 184 & 142 & \\
\hline & \multicolumn{3}{|c|}{ PNG AN018 } & 149 & 174 & SMNTHALSQDLR & 328 & 192 & 139 & \\
\hline
\end{tabular}

Note: Boldface amino acids represent the mutated state of that codon.

${ }^{\mathrm{a}} \mathrm{MS}$ microsatellite upstream of pfcrt:B5M77 $(-20 \mathrm{~kb})$ and 2E10 (-5 kb).

${ }^{\mathrm{b}}$ Amino acid (AA) positions in pfcrt are 72, 74, 75, 76, 97, 144, 160, 220, 271, 326, 356, 371.

${ }^{C} \mathrm{MS}$ microsatellite downstream of pfcrt: PE12A $(+6 \mathrm{~kb}), 2 \mathrm{H} 4(+22 \mathrm{~kb})$ and PE14F $(+106 \mathrm{~kb})$.

${ }^{\mathrm{d}}$ Amino acid positions in pfmdr1 are 86, 184, 1034, 1042, 1246.

*not determined.

** or *** indicates 2 or 3 AA positions were not determined 
Table 3 Prevalence of MS haplotypes between different island groups

\begin{tabular}{cccccccc}
\hline \multirow{2}{*}{ Country } & Province & \multicolumn{6}{c}{ Prevalence (\%) } \\
\cline { 3 - 8 } & & H1 & H2 & H3 & H4 & H5 & H6 \\
\hline Solomon Islands & Temotu & 0.0 & 0.0 & 76.5 & 20.6 & 2.9 & 0.0 \\
& Malaita & 7.1 & 0.0 & 71.4 & 14.3 & 0.0 & 7.1 \\
\multirow{2}{*}{ Vanuatu } & Tafea & 75.0 & 25.0 & 0.0 & 0.0 & 0.0 & 0.0 \\
\hline
\end{tabular}

possessing a variation at an upstream locus compared to $\mathrm{H} 3$ and H4. One sample was determined H6, also having a variation at an upstream locus.

In Tanna Island, Vanuatu, eight of 17 isolates were successfully typed at all five loci resulting in two patterns (based on similarity of 4/5 MS markers). Of these $75 \%$ (6/8) were determined as H1 (Table 3) showing close identity to those observed for the Solomon N70 group [12]. The two remaining samples, from the village of Fetukai, had unique MS sizes at three loci, while locus PE14F was observed to be identical to samples from the Thailand $\mathrm{C} 2 \mathrm{~B}$ group. These two samples were classified as H2. Interestingly, despite having the SMNTHAL in PfCRT, they also possessed a change in amino acid from glutamine $(\mathrm{Q})$ to glutamic acid (E) at codon 271, a characteristic common to both groups of samples originating from Thailand (Dd2 and C2B).

\section{Prevalence of mutations in pfmdr1}

Sequences of $p f m d r 1$ were analysed and the prevalence of mutations in the three island groups determined (Table 4). Amino acid substitutions were identified at codons 86, 184 and 1034 while only wild type codons were observed at 1042 and 1246. Polymorphisms N86Y and S1034C were present in $100 \%$ of samples from both countries, while one sample from Vanuatu possessed an additional mutation Y184F. The dominant $p f m d r 1$ allele across all island groups was YYCND. The observed allelic types in the different island groups are summarized in Table 2.

\section{Discussion}

CQ has been used widely in the South Pacific for many years and has applied a strong selection pressure on

\begin{tabular}{|c|c|c|c|c|c|c|c|}
\hline \multirow[t]{2}{*}{ Country } & \multirow[t]{2}{*}{ Province } & \multirow[t]{2}{*}{$\mathrm{n}$} & \multicolumn{5}{|c|}{$\begin{array}{l}\text { \% substitutions at amino acid } \\
\text { positions in PfMDR1 }\end{array}$} \\
\hline & & & 86 & 184 & 1034 & 1042 & 1246 \\
\hline Vanuatu & Tafea & 17 & 100 & 6 & 100 & 0 & 0 \\
\hline \multirow[t]{2}{*}{ Solomon Islands } & Temotu & 37 & 100 & 0 & 100 & 0 & 0 \\
\hline & Malaita & 31 & 100 & 0 & 100 & 0 & 0 \\
\hline
\end{tabular}

PfMDR1 = Plasmodium falciparum multidrug resistance protein 1. parasite populations, which may affect the efficacy of ACT. Vanuatu and the Solomon Islands are among the few countries where ACT is currently used for treatment of both $P$. falciparum and $P$. vivax malaria. Understanding the parasite drug resistance profile at the time of ACT introduction allows for detection of changes in future parasite populations. It will also enable a better understanding of potential changes in drug resistance profiles for both species in the absence of CQ drug pressure. In this paper, the baseline data on pfcrt and pfmdr1 was investigated at the time of AL introduction.

Overall results of this study indicated a dominance of the SMNT allele across all island groups. Amino acid analysis indicated $100 \%$ of samples examined from Tafea Province, Vanuatu and Malaita Province, Solomon Islands, and $98.8 \%$ of samples from Temotu Province, Solomon Islands possessed the K76T polymorphism indicative of CQ resistance, and possibly impaired quinine and halofantrine susceptibility. This also demonstrates that the CQR pfcrt allele is at fixation in these three island group populations of Vanuatu and Solomon Islands. The high prevalence of CQR parasites in these two countries is not surprising given $\mathrm{CQ}$ monotherapy was the primary chemotherapeutic drug used to treat uncomplicated $P$. falciparum malaria for several decades, and until relatively recently, was also the treatment of choice for $P$. vivax infections. Although $\mathrm{CQ}$ has not been used in Vanuatu and the Solomon Islands as a monotherapy since 1994 and 1991, respectively, it was still used in combination therapy for treatment of $P$. vivax infections. This continued CQ pressure provides a reasonable explanation for the prevalence of the $76 \mathrm{~T}$ mutation in these parasite populations. Interestingly, published in vivo and in vitro studies suggest the effectiveness of lumefantrine is enhanced in P. falciparum parasites carrying the pfcrt K76T mutation [13]. This suggests that lumefantrine is an ideal ACT partner drug to use in Vanuatu and the Solomon Islands as both these regions exhibit high levels of $C Q$ resistance.

All samples exhibiting the K76T polymorphism also possessed additional mutations occurring in at least one of the following codons 220,326, 356, and 371. While SMNTHALSQDLR was predominant on all three island groups, variation in pfcrt genotypes exists not only between these two countries but also within island groups. In Temotu Province, one of 50 samples analysed retained a wild type $\mathrm{K} 76$ in conjunction with a single A220S polymorphism (genotype CMNKHALSQNIR). Although the A220S polymorphism in pfcrt has been shown to increase the level of CQ resistance when co-existing with $\mathrm{K} 76 \mathrm{~T}$, it is unlikely that a single A220S polymorphism is sufficient to confer CQR [28]. In contrast, another isolate from the village of Ngamubulo situated on the Reef Islands did not exhibit the A220S substitution generally associated with 
CQR alleles having a genotype of SMNTH ${ }^{* * *} \mathrm{~A}^{* * T R}$. The occurrence of the A220 in combination with the K76T mutation, although not common, has been previously documented in isolates from the Philippines, as well as India and China [12,29,30]. $\mathrm{Q}$ is found at codon 271 in all samples, including reference samples, except those originating in Thailand, which predominantly contain an $\mathrm{E}$ at this position. Interestingly, $43 \%$ of samples from Tafea Province, Vanuatu contained the Q271E substitution. At this point the drug pressure that selects for this polymorphism is not understood; however, it may be indicative of changes in drug sensitivity profiles and/or the subsequent sweep of drug resistant alleles throughout the region, hence this codon may be a key amino acid for future surveillance studies.

Overall, results indicated that $74.5 \%(38 / 51)$ of samples collected from Tafea Province, Vanuatu and Temotu Province, Solomon Islands, for which all 12 pfcrt codons could be determined, had a pfcrt allelic type identical to those from Papua New Guinea (PNG). This may indicate a gene flow of parasite alleles between these island groups and suggests that malaria drug resistance in these provinces is similar to PNG. At this time it is unclear if clinical drug resistance differences exist between Tafea Province, Vanuatu and Temotu Province, Solomon Islands, however $p f c r t$ analysis of $P$. falciparum samples collected from these locations predict similar drug susceptibilities. Furthermore, the SMNTH allelic type of pfcrt in these island groups suggests a past parasite exposure to amodiaquine (AQ) [7] although no evidence of AQ use in either Vanuatu or the Solomon Islands is evident. AQ was however introduced in PNG in 1966 and given its close geographical proximity, the likelihood of drug resistance genotypes becoming established in the neighbouring countries of Solomon Islands and Vanuatu seems predictable.

Analysis of five MS markers flanking pfcrt was undertaken to determine whether parasites harbouring mutations that conferred $\mathrm{CQR}$ evolved locally or originated via gene flow from other countries. Overall, 70 of 98 samples from three island groups were typed revealing six MS haplotypes. There were difficulties in obtaining MS data for many of the samples analysed, particularly from Tanna Island and Temotu Province; this was possibly due to low levels of parasitaemia in these samples as these were obtained from cross-sectional surveys of villagers most of whom were asymptomatic and had low parasitaemia at time of survey. Interestingly, also in Tafea and Temotu Provinces there was a high incidence of asymptomatic infections. In Tafea Province only $28 \%$ of children that were slide positive for malaria were febrile while in Temotu Province only $14 \%$ of all ages tested that were slide positive for malaria were febrile [31]. This suggests in these regions that a high proportion of people infected with malaria were asymptomatic. In comparison $63 \%$ of samples collected from Malaita Province were from febrile patients attending the clinic or hospital. This implies that only sampling from symptomatic individuals (ie. those attending clinics or hospitals) has the potential to miss many positive malaria cases and this may lead to undetected genotypes circulating within populations.

Interestingly, the distribution and prevalence of the six haplotypes were different between the island groups. H3 and $\mathrm{H} 4$ were predominant haplotypes in both Temotu and Malaita Provinces, Solomon Islands. Both these haplotypes were also dominant types in PNG $[10,12]$. The MS and the pfcrt typing results strongly indicate that CQR parasites in two different provinces of Solomon Islands share the same origin as those from PNG and may have resulted from a spread via PNG. Minor haplotypes were also observed in these two island groups, $\mathrm{H} 5$ was present in Temotu while H1 and H6 were in Malaita Province. H5 has a distinct MS pattern upstream to $p f c r t$ and has a wild type $p f c r t$ representing a minor CQsensitive parasite population. The minor types $\mathrm{H} 1$ and $\mathrm{H} 6$, have small variations to $\mathrm{H} 3$ and $\mathrm{H} 4$, and may have resulted from genetic drift.

In Tafea Province, Vanuatu, H1 appears to be the dominant haplotype albeit the sample number is relatively small. Although there is variation, it is closely related to $\mathrm{H} 4$, which has been reported in samples from Guadalcanal, Solomon Islands previously [12]. Therefore, parasites in this province may have spread from Solomon Islands or PNG. The haplotype H2 $(n=2)$ observed in this location may have been generated from a recombination between Southeast Asian and PNG parasites. The Q271E polymorphism detected in these two parasites, which is common to Southeast Asian CQR parasites, and the presence of Southeast Asian CQR pfcrt allele in the province support this hypothesis.

Interactions between $p f c r t$ and $p f m d r 1$ mutations and their combined effect on drug resistance are not clear. Like $p f c r t$, direct evidence has demonstrated that point mutations in pfmdrl can confer resistance to CQ, mefloquine, quinine, and halofantrine [15]. It has been suggested that the response of $C Q R$ falciparum parasites to $\mathrm{CQ}$ is associated not only with the pfcrt genotype but also the $p f m d r 1$ mutations with which they are coupled [7]. In this study the dominant (98.5\%) pfmdr1 allele across all island groups was YYCND, only one isolate from Vanuatu possessed a YF*ND allele. Studies have suggested that the Y184F mutation is associated with lowlevel resistance to AL [32] but as yet there has been no consensus for the role it plays in CQ resistance. The change in amino acid from asparagine to tyrosine N86Y has been associated with CQ resistance in some investigations [33-36] but not others [37-40]; this polymorphism has also been implicated in playing a modulating role in reducing the susceptibility of parasites to amodiaquine 
[41-44], a 4-aminoquinoline drug structurally related to CQ. A clinical trial in Zanzibar, in which pfcrt K76T and pfmdr1 N86Y frequencies were determined before $\mathrm{AL}$ administration and then again in all recurrent parasites during a follow-up period of 42 days, showed a significant increase in the occurrence of $p f m d r 1 \mathrm{~N} 86$, suggesting N86 as a potential marker of lumefantrine resistance in vivo [45]. Thus, future surveillance studies in Vanuatu and the Solomon Islands, post implementation of AL, will be of interest to see if there are similar changes in the frequency of $p f m d r 1$ mutations.

\section{Conclusions}

This study provides a clear indication of widespread CQ resistance in both the Solomon Islands and Vanuatu at the time of AL introduction. Overall the pfcrt genotypes and the MS markers flanking them in samples from Tafea Province, Vanuatu, and Temotu and Malaita Provinces, Solomon Islands exhibited similarities to those of PNG suggesting that malaria drug resistance profiles are also similar between these countries. The recent introduction and use of AL throughout this region as first-line treatment for both $P$. falciparum and $P$. vivax infections, warrants continued surveillance not only to monitor baseline but also to observe correlative changes in the pfcrt allele in the absence of CQ pressure.

\section{Competing interests}

The authors declare that they have no competing interests.

\section{Authors' contributions}

KJG and KG performed experiments determining parasite species and genotypes; KJG and QC carried out data analysis; $A B$ and GT contributed to the baseline survey; JH and LW contributed to the therapeutic efficacy studies, KJG and QC wrote the manuscript; KJG, NW and QC conceived and designed the study. All authors read and approve the final manuscript.

\section{Acknowledgements}

We thank the Ministries of Health Solomon Islands and Vanuatu for support and approval to conduct these studies. We thank the people of the Solomon Islands and Vanuatu for their hospitality and willingness to participate in the malaria survey. We are grateful to Dr Bridget Appleyard and Dr Dorina Bustos for facilitating the therapeutic efficacy studies and standardizing the filter paper preparation in Malaita. Funding for the malaria survey was provided by AusAID through Pacific Malaria Initiative Supporting Centre (PacMISC), University of Queensland. Genotyping analysis and drug resistance profiling was financially supported by Department of Defense, Global Emerging Infections Surveillance Program (DoD-GEIS), USA.

\section{Disclaimer}

The opinions expressed herein are those of the authors and do not necessarily reflect those of the Australian Defence Force Joint Health Command or the US Department of Defense.

\section{Author details}

${ }^{1}$ Australian Army Malaria Institute, Enoggera, Brisbane, Queensland, Australia. ${ }^{2}$ QIMR Berghofer Medical Research Institute, Brisbane, Queensland, Australia. ${ }^{3}$ Malaria and Vector Borne Diseases Control Program, Ministry of Health, Honiara, Solomon Islands. ${ }^{4}$ Vector Borne Disease Control Program, Ministry of Health, Port Vila, Vanuatu. ${ }^{5}$ School of Public Health, Tropical Medicine and Rehabilitation Sciences, James Cook University, Townsville, Queensland, Australia. ${ }^{6}$ Walter Reed Army Institute of Research, Malaria Vaccine Branch, Military Malaria Research Program, Silver Spring, Maryland, USA.
Received: 18 July 2014 Accepted: 7 October 2014

Published: 15 October 2014

\section{References}

1. Zucker JR, Lackritz EM, Ruebush TK, Hightower AW, Adungosi JE, Were JB, Metchock B, Patrick E, Campbell CC: Childhood mortality during and after hospitalization in western Kenya: effect of malaria treatment regimens. Am J Trop Med Hyg 1996, 55:655-660.

2. Trape JF, Pison G, Preziosi MP, Enel C, Desgrees-du LA, Delaunay V, Samb B, Lagarde E, Molez JF, Simondon F: Impact of chloroquine resistance on malaria mortality. CR Acad Sci III 1998, 321:689-697.

3. WHO: Antimalarial drug combination therapy. Report of a WHO technical consultation. Geneva: World Health Organization, Geneva; 2001.

4. WHO: World Malaria Report 2013. Geneva: World Health Organization; 2013.

5. Fidock DA, Nomura T, Talley AK, Cooper RA, Dzekunov SM, Ferdig MT, Ursos LM, Sidhu AB, Naude B, Deitsch KW, Su XZ, Wootton JC, Roepe PD, Wellems TE: Mutations in the $P$. falciparum digestive vacuole transmembrane protein PfCRT and evidence for their role in chloroquine resistance. Mol Cell 2000, 6:861-871.

6. Sidhu $A B$, Verdier-Pinard D, Fidock DA: Chloroquine resistance in Plasmodium falciparum malaria parasites conferred by pfcrt mutations. Science 2002, 298:210-213.

7. Sa JM, Twu O, Hayton K, Reyes S, Fay MP, Ringwald P, Wellems TE: Geographic patterns of Plasmodium falciparum drug resistance distinguished by differential responses to amodiaquine and chloroquine. Proc Natl Acad Sci U S A 2009, 106:18883-18889.

8. Wellems TE, Plowe CV: Chloroquine-resistant malaria. J Infect Dis 2001, 184:770-776.

9. Wootton JC, Feng X, Ferdig MT, Cooper RA, Mu J, Baruch DI, Magill AJ, Su $X Z$ : Genetic diversity and chloroquine selective sweeps in Plasmodium falciparum. Nature 2002, 418:320-323.

10. Mehlotra RK, Fujioka H, Roepe PD, Janneh O, Ursos LM, Jacobs-Lorena V, McNamara DT, Bockarie MJ, Kazura JW, Kyle DE, Fidock DA, Zimmerman PA: Evolution of a unique Plasmodium falciparum chloroquine-resistance phenotype in association with pfcrt polymorphism in Papua New Guinea and South America. Proc Natl Acad Sci USA 2001, 98:12689-12694.

11. Chen N, Kyle DE, Pasay C, Fowler EV, Baker J, Peters JM, Cheng Q: pfcrt allelic types with two novel amino acid mutations in chloroquineresistant Plasmodium falciparum isolates from the Philippines. Antimicrob Agents Chemother 2003, 47:3500-3505.

12. Chen N, Wilson DW, Pasay C, Bell D, Martin LB, Kyle D, Cheng Q: Origin and dissemination of chloroquine-resistant Plasmodium falciparum with mutant pfcrt alleles in the Philippines. Antimicrob Agents Chemother 2005, 49:2102-2105.

13. Sisowath C, Petersen I, Veiga MI, Martensson A, Premji Z, Bjorkman A, Fidock DA, Gil JP: In vivo selection of Plasmodium falciparum parasites carrying the chloroquine-susceptible pfcrt K76 allele after treatment with artemether-lumefantrine in Africa. J Infect Dis 2009, 199:750-757.

14. Foote SJ, Kyle DE, Martin RK, Oduola AM, Forsyth K, Kemp DJ, Cowman AF: Several alleles of the multidrug-resistance gene are closely linked to chloroquine resistance in Plasmodium falciparum [see comments]. Nature 1990, 345:255-258.

15. Reed MB, Saliba KJ, Caruana SR, Kirk K, Cowman AF: Pgh1 modulates sensitivity and resistance to multiple antimalarials in Plasmodium falciparum. Nature 2000, 403:906-909.

16. Duraisingh MT, Cowman AF: Contribution of the pfmdr1 gene to antimalarial drug-resistance. Acta Trop 2005, 94:181-190.

17. Sidhu $A B$, Valderramos SG, Fidock DA: pfmdr 1 mutations contribute to quinine resistance and enhance mefloquine and artemisinin sensitivity in Plasmodium falciparum. Mol Microbiol 2005, 57:913-926.

18. Price RN, Cassar C, Brockman A, Duraisingh M, Van Vugt M, White NJ, Nosten F, Krishna S: The pfmdr1 gene is associated with a multidrug-resistant phenotype in Plasmodium falciparum from the western border of Thailand. Antimicrob Agents Chemother 1999, 43:2943-2949.

19. Sidhu AB, Uhlemann AC, Valderramos SG, Valderramos JC, Krishna S, Fidock DA: Decreasing pfmdr1 copy number in Plasmodium falciparum malaria heightens susceptibility to mefloquine, lumefantrine, halofantrine, quinine, and artemisinin. J Infect Dis 2006, 194:528-535.

20. Chavchich M, Gerena L, Peters J, Chen N, Cheng Q, Kyle DE: Role of pfmdr1 amplification and expression in induction of resistance to artemisinin 
derivatives in Plasmodium falciparum. Antimicrob Agents Chemother 2010, 54:2455-2464.

21. Bowden DK, Bastien P, Douglas FP, Muir JW, Tambisari E: Chloroquine-resistant Plasmodium falciparum malaria in Vanuatu. Med $\mathrm{J}$ Aust 1982, 2:561-562.

22. WHO: Review of the malaria drug efficacy situation in 10 countries of the WHO Western Pacific Region 1987-2003. Geneva: World Health Organization; 2005.

23. The Pacific Malaria Inititive Survey Group: Malaria on isolated Melanesian islands prior to the initiation of malaria elimination activities. Malar J 2010, 9:218.

24. Padley D, Moody AH, Chiodini PL, Saldanha J: Use of a rapid, single-round, multiplex PCR to detect malarial parasites and identify the species present. Ann Trop Med Parasitol 2003, 97:131-137.

25. Djimde A, Doumbo OK, Cortese JF, Kayentao K, Doumbo S, Diourte $Y$, Dicko A, Su XZ, Nomura T, Fidock DA, Wellems TE, Plowe CV, Coulibaly D: A molecular marker for chloroquine-resistant falciparum malaria. $N$ Engl $J$ Med 2001, 344:257-263.

26. Chen N, Russell B, Staley J, Kotecka B, Nasveld P, Cheng Q: Sequence polymorphisms in pfcrt are strongly associated with chloroquine resistance in Plasmodium falciparum. J Infect Dis 2001, 183:1543-1545.

27. Chen N, Russell B, Fowler E, Peters J, Cheng Q: Levels of chloroquine resistance in Plasmodium falciparum are determined by loci other than pfcrt and pfmdr1. J Infect Dis 2002, 185:405-407.

28. Summers RL, Dave A, Dolstra TJ, Bellanca S, Marchetti RV, Nash MN, Richards SN, Goh V, Schenk RL, Stein WD, Kirk K, Sanchez CP, Lanzer M, Martin RE: Diverse mutational pathways converge on saturable chloroquine transport via the malaria parasite's chloroquine resistance transporter. Proc Natl Acad Sci U S A 2014, 111:E1759-E1767.

29. Mittra P, Vinayak S, Chandawat H, Das MK, Singh N, Biswas S, Dev V, Kumar A, Ansari MA, Sharma YD: Progressive increase in point mutations associated with chloroquine resistance in Plasmodium falciparum isolates from India. J Infect Dis 2006, 193:1304-1312.

30. Yang Z, Zhang Z, Sun X, Wan W, Cui L, Zhang X, Zhong D, Yan G: Molecular analysis of chloroquine resistance in Plasmodium falciparum in Yunnan Province, China. Trop Med Int Health 2007, 12:1051-1060.

31. Harris I, Sharrock WW, Bain LM, Gray KA, Bobogare A, Boaz L, Lilley K Krause D, Vallely A, Johnson ML, Gatton ML, Shanks GD, Cheng Q: A large proportion of asymptomatic Plasmodium infections with low and sub-microscopic parasite densities in the low transmission setting of Temotu Province. Solomon Islands: challenges for malaria diagnostics in an elimination setting. Malar J 2010, 9:254.

32. Sisowath C, Ferreira PE, Bustamante LY, Dahlstrom S, Martensson A Bjorkman A, Krishna S, Gil JP: The role of pfmdr1 in Plasmodium falciparum tolerance to artemether-lumefantrine in Africa. Trop Med Int Health 2007, 12:736-742.

33. Babiker HA, Pringle SJ, bdel-Muhsin A, Mackinnon M, Hunt P, Walliker D: High-level chloroquine resistance in Sudanese isolates of Plasmodium falciparum is associated with mutations in the chloroquine resistance transporter gene pfcrt and the multidrug resistance Gene pfmdr1. J Infect Dis 2001, 183:1535-1538.

34. Basco LK, Le-Bras J, Rhoades Z, Wilson CM: Analysis of pfmdr1 and drug susceptibility in fresh isolates of Plasmodium falciparum from subsaharan Africa. Mol Biochem Parasitol 1995, 74:157-166.

35. Cojean S, Noel A, Garnier D, Hubert V, Le BJ, Durand R: Lack of association between putative transporter gene polymorphisms in Plasmodium falciparum and chloroquine resistance in imported malaria isolates from Africa. Malar J 2006, 5:24

36. Duraisingh MT, Jones $P$, Sambou I, Von Seidlein L, Pinder M, Warhurst DC: The tyrosine-86 allele of the pfmdr1 gene of Plasmodium falciparum is associated with increased sensitivity to the anti-malarials mefloquine and artemisinin. Mol Biochem Parasitol 2000, 108:13-23.

37. Awad-el KFM, Miles MA, Warhurst DC: Chloroquine-resistant Plasmodium falciparum isolates from the Sudan lack two mutations in the pfmdr 1 gene thought to be associated with chloroquine resistance. Trans $R$ Soc Trop Med Hyg 1992, 86:587-589.

38. Basco LK, Ringwald P: Molecular epidemiology of malaria in Cameroon. $\mathrm{X}$. Evaluation of PFMDR1 mutations as genetic markers for resistance to amino alcohols and artemisinin derivatives. Am J Trop Med Hyg 2002, 66:667-671.

39. Flueck TP, Jelinek T, Kilian AH, Adagu IS, Kabagambe G, Sonnenburg F, Warhurst DC: Correlation of in vivo-resistance to chloroquine and allelic polymorphisms in Plasmodium falciparum isolates from Uganda. Trop Med Int Health 2000, 5:174-178.

40. Grobusch MP, Adagu IS, Kremsner PG, Warhurst DC: Plasmodium falciparum: in vitro chloroquine susceptibility and allele-specific PCR detection of Pfmdr1 Asn86Tyr polymorphism in Lambarene, Gabon. Parasitology 1998, 116:211-217.

41. Happi CT, Gbotosho GO, Folarin OA, Bolaji OM, Sowunmi A, Kyle DE, Milhous W, Wirth DF, Oduola AM: Association between mutations in Plasmodium falciparum chloroquine resistance transporter and $P$. falciparum multidrug resistance 1 genes and in vivo amodiaquine resistance in P. falciparum malaria-infected children in Nigeria. Am J Trop Med Hyg 2006, 75:155-161.

42. Djimde AA, Fofana B, Sagara I, Sidibe B, Toure S, Dembele D, Dama S, Ouologuem D, Dicko A, Doumbo OK: Efficacy, safety, and selection of molecular markers of drug resistance by two ACTs in Mali. Am J Trop Med Hyg 2008, 78:455-461.

43. Holmgren G, Gil JP, Ferreira PM, Veiga Ml, Obonyo CO, Bjorkman A: Amodiaquine resistant Plasmodium falciparum malaria in vivo is associated with selection of pfcrt $76 \mathrm{~T}$ and pfmdr1 86Y. Infect Genet Evol 2006, 6:309-314.

44. Holmgren G, Hamrin J, Svard J, Martensson A, Gil JP, Bjorkman A: Selection of pfmdr1 mutations after amodiaquine monotherapy and amodiaquine plus artemisinin combination therapy in East Africa. Infect Genet Evol 2007, 7:562-569.

45. Sisowath C, Stromberg J, Martensson A, Msellem M, Obondo C, Bjorkman A, Gil JP: In vivo selection of Plasmodium falciparum pfmdr1 $86 \mathrm{~N}$ coding alleles by artemether-lumefantrine (Coartem). J Infect Dis 2005, 191:1014-1017.

doi:10.1186/1475-2875-13-406

Cite this article as: Gresty et al:: Genetic mutations in pfort and pfmdr 1 at the time of artemisinin combination therapy introduction in South Pacific islands of Vanuatu and Solomon Islands. Malaria Journal 2014 13:406

\section{Submit your next manuscript to BioMed Central and take full advantage of:}

- Convenient online submission

- Thorough peer review

- No space constraints or color figure charges

- Immediate publication on acceptance

- Inclusion in PubMed, CAS, Scopus and Google Scholar

- Research which is freely available for redistribution 